\title{
La dimensión democrática de la nueva gestión pública
}

\section{Quim Brugué *}

El eje temático de este nuevo número de la revista GAPP ha estado injustamente marginado de los apasionados debates que ha suscitado la modernización de la administración pública. La atracción que ha ejercido la gestión empresarial, las modas privatizadoras y desreguladoras o, más genéricamente, el desprestigio del gobierno y de la cosa pública contribuyen a explicar por qué, durante la última década, la dimensión democrática de la administración pública ha aparecido únicamente en algunas eruditas notas a pie de página. Esta marginación, sin embargo, no ha conseguido reducir la importancia del tema. Ha conseguido, en cambio, distorsionar peligrosamente el discurso de la modernización administrativa.

Referirse a la dimensión democrática de la administración pública no supone un menoscabo de algo que sí ha estado muy presente en los debates recientes sobre su modernización: la mejora en la economía y la eficiencia de sus actuaciones. Supone, en cambio, recordar que la administración pública no se entiende sin sus referentes políticos y sociales. En otras palabras, supone reconocer que la administración pública es algo más que un instrumento técnico, un brazo ejecutivo o un mecanismo dedicado inocuamente a lo que podríamos denominar una implementación aséptica de órdenes o directrices emanadas en centros políticos de decisión. Muy al contrario, la administración pública configura un espacio donde surgen y se resuelven conflictos, donde interactúan intereses contrapuestos, donde se prioriza entre valores en competencia y donde, en definitiva, se dan cita todos los elementos de un debate político.

Además, recuperar la dimensión democrática de la administración pública supone también aceptar que los conflictos, las luchas, los valores o los intereses que se entrecruzan en su interior no deben resolverse - al menos de forma exclusiva- a través de mecanismos mercantiles. El mercado sirve para regular los conflictos y las relaciones entre individuos que intentan maximizar sus beneficios, y puede ser muy eficiente en este terreno. La administración pública debe orientarse a la configuración de un determinado modelo de sociedad, sus actuaciones sirven para darle una forma determinada y, por lo tanto, incorpora una elección no exenta de conflictividad. Sin embargo, regular esta conflictividad y dar con el modelo de sociedad que pretende fomentar la administración no es tarea de un mercado competitivo donde los grupos o los individuos intentan imponer sus preferencias, sino de la expresión de voluntades colectivas. Voluntades colectivas que, en nuestro sistema político, han de expresarse democráticamente.

De este modo, el déficit democrático al que me refería inicialmente debe entenderse como la incapacidad de las administraciones públicas para articular un discurso político - un debate democrático- que sirva para resolver los conflictos que se dan en su interior, para priorizar entre valores en competencia $y$, en definitiva, para permitir al conjunto social escoger para qué diablos quiere su administración. La modernización de la administración pública parece recuperar la distincion wilsoniana entre decisión política y ejecución administrativa, lo cual le permite centrarse en la mejor forma de llevar a término su especialidad ejecutora. Lo que aquí sugiero es que se trata de una distinción falaz y que, si reconocemos que la realidad administrativa configura un potaje donde es prácticamente imposible distinguir sus ingredientes políticos y técnicos, entonces debería preocuparnos su democratización. Es decir, deberíamos reflexionar sobre cómo articular mecanismos para controlar democráticamente por qué nuestras administraciones hacen una cosa y no otra, por qué priorizan unas actuaciones y no otras, por qué escuchan más a unos actores sociales que a otros, por qué promocionan unos valores y no otros.

En este artículo, por lo tanto, no pretendo analizar detalladamente las características de la modernización de la administración pública, sino algo mucho más limitado: leer las notas a pie de página de algunos de sus teóricos más relevantes y, así, observar qué nos dicen $\multimap$, mejor, que intuyo que nos dicensobre la dimensión democrática de la nueva gestión pública, de la posburocracia, del gobierno reinventado o de como prefiramos llamar a cualquiera de los intentos de modificación de la administración pública tradicional. No se trata de un análisis 
académico, sino más bien de una reflexión muy personal realizada en voz alta. Es por esta razón que he optado por una fórmula expositiva más relajada, sin sentirme obligado a usar constantes referencias bibliográficas y persiguiendo más la capacidad para suscitar la discusión que la rigurosidad científica.

Con estos objetivos, he estructurado el artículo en cinco apartados. En el primero esbozo los grandes rasgos del modelo tradicional de administración (en su expresión weberiana más pura), así como los de su crisis y los de la emergente nueva gestión pública. No lo hago de forma exhaustiva, sino intentando subrayar los déficit democráticos del primer modelo y observando como su reformulación abre nuevos interrogantes sobre estos mismos déficit. Interrogantes que, a su vez, suscitan respuestas diferentes en la literatura más reciente sobre la nueva gestión pública.

En el marco de esta nueva gestión pública, he identificado un mínimo de tres posturas relacionadas con la dimensión democrática de la administración pública, las cuales resumo en los apartados dos, tres y cuatro. En el segundo me refiero a lo que considero las posiciones más crédulas; es decir, las de aquellos que responden a nuestro interrogante desde el optimismo, confiando en la capacidad de la emergente posburocracia para superar los problemas de eficiencia de la burocracia y, en buena medida, olvidando los déficit democráticos que ésta plantea. En el tercer apartado, en cambio, repaso algunos planteamientos más desconfiados. Bajo el epígrafe del neoweberianismo aparecen una serie de autores que no creen que la posburocracia aporte ninguna idea sustancialmente diferente, sino que más bien se trata de un afianzamiento de la propia burocracia y que, por lo tanto, reproduce $\longrightarrow$ incluso intensifica- su déficit democrático. En el cuarto apartado me acerco a las posturas más criticas; es decir, a aquellas aportaciones que subrayan los peligros de regresión democrática asociados a los procesos de modernización de la administración pública. Finalmente, en el último apartado, intentaré recapitular el debate y, en la medida de las fuerzas que me queden, fijar mi posición al respecto.

\section{El punto de partida}

A través de una imagen muy afortunada, una estudioso de la administración pública como Hood convierte el mundo occidental en un parque jurásico dónde aparecen y desaparecen los grandes dinosaurios que definen sus reglas de funcionamiento. En su libro, Explaining Economic Policy Reversals, Hood se pregunta por qué los grandes dinosaurios de las políticas públicas de los años cincuenta y sesenta se han extinguido, de forma relativamente incomprensible, durante la década de los ochenta. Más concretamente, se pregunta por qué la gran empresa pública ha sido sustituida por procesos de privatización, por qué las políticas keynesianas han dejado paso a las políticas monetaristas, por qué la voluntad reguladora ha desaparecido frente a las modas desreguladoras o, entre otras cosas, por qué la administración pública tradicional (APT) ha cedido ante la presión de la nueva gestión pública (NGP). Este último punto es el que ahora nos interesa.

El viejo dinosaurio de la APT se había impuesto a la más primitiva especie de la administración pública pre-moderna gracias a su capacidad para actuar con mayor competencia y para eliminar la corrupción de un modelo construido sobre lealtades personales y favoritismos. Capacidades que había desarrollado abrevando en dos manantiales: el weberianismo y el taylorismo. Situados en el sector público, el primer manantial es el que nos ofrece un mayor caudal.

Max WeBer desarrolló el concepto de burocracia para referirse a unas organizaciones jerárquicas donde los individuos tenían unas responsabilidades estrictamente definidas y donde sus actuaciones estaban reguladas a través de reglas escritas y de mecanismos de autoridad vertical. El modelo burocrático es la expresión del proceso de creación de la modernidad occidental, de una modernidad racional que -en palabras de Ritzer - se manifiesta en el dominio de la eficiencia, de la predictibilidad, de la calculabilidad y del control de los individuos a través de la tecnología. En opinión de WEBER, la racionalidad formal implica que cuando los individuos buscan los medios óptimos para lograr determinados fines, éstos disponen de unas normas, de unas regulaciones y de unas instituciones sociales que predefinen este óptimo. No es necesario descubrirlo individualmente, pues el óptimo ya ha sido primero descubierto y, más tarde, institucionalizado a través de reglas, procedimientos y estructuras. La gente sólo ha de seguir las normas, dejarse llevar por los mandatos de la razón institucionalizada.

Evidentemente, los supuestos weberianos han ido evolucionando. No es lo mismo la burocracia de finales del siglo XIX que la de mediados del siglo XX, ya que ha ido adaptándose a los requisitos de cada momento. En cualquier caso, se detecta una línea de continuidad relacionada con su vocación racionalizadora, aunque ésta se manifieste primero a través de la juridificación y, más tarde, de la tecnificación.

Dejando al margen este proceso de cambio interno, la principal diferencia entre la burocracia y otras formas organizativas pre-modernas radica en su mayor eficiencia. La institucionalización de determinadas reglas y procedimientos no sólo nos conduce sino que incluso nos fuerza a ser eficientes, a utilizar los medios óptimos en la consecución de los fines. Pero el énfasis en la eficiencia puede resultar incluso excesivo $y$, tal como suguiere TAKAKI, puede generar lo que se conoce como sla irracionalidad de la racionalidad. Es decir, un proceso global de racionalización puede generar un mundo donde la gente no sea capaz de comportarse como un ser humano, don- 
de se produzca su total deshumanización. En un libro tan interesante como Modernity and the Holocaust, BaUman analiza el holocausto nazi como la peor de las expresiones de la racionalización, como la representación más extrema de ala irracionalidad de la racionalidad, como el más acabado de los procesos de deshumanización. En este sentido, el holocausto nazi se orquestó sobre los rasgos definidores de la racionalidad formal: se trataba de un mecanismo eficiente para el asesinato masivo; dotado de calculabilidad, pues se trataba de mejorar el ratio muerte/tiempo; con voluntad de predictabilidad, ya que organizaba las matanzas siguiendo el esquema casuístico de la producción en cadena; $y$, finalmente, el sistema de ferrocarriles, los crematorios o la burocracia que organizaba el proceso representaban el control a través de unas tecnologias deshumanizadas, de unos procesos técnicamente impolutos.

Los efectos perversos de la racionalidad burocrática están relacionados con su déficit democrático. Este es el tema que nos ocupa, aunque después de la lectura del libro de Bauman estos déficit nos puedan parecer ridículos.

Utilizando las síntesis de autores como Hood y Hughes, podemos afirmar que la APT presentaba dos grandes problemas. En primer lugar, la propia eficiencia del modelo burocrático se veía afectada por el excesivo celo respecto a las reglas y los procedimientos. En segundo lugar, la siempre falaz separación entre políticos y admininistradores, la pretendida despolitización de la burocracia, la deshumanización de los procesos racionalizadores o las propias condiciones de trabajo que imponía el modelo generaban dificultades en la tarea de control político. Es decir, la burocracia se ha mostrado en constante fricción con la democracia: con una democracia basada en unos individuos a los que la racionalización despoja de su condición de seres humanos, y con una democracia basada en una capacidad de elección que desaparece una vez la racionalidad ha intitucionalizado los comportamientos óptimos. Si deshumanizamos y limitamos la capacidad de elección de los individuos, ¿no sufre con ello la propia esencia de la democracia?

Siguiendo este esquema, Hood afirma que la pérdida de eficiencia y las fricciones con la democracia son los dos fenómenos que desencadenan la sustitución del viejo dinosaurio de la APT por el nuevo dinosaurio de la NGP. Una NGP que se construye a partir de la revisión de los dos pilares que ya había revisado la propia APT. Por un lado, la competencia no se consigue únicamente a través de reglas y procedimientos, sino que reclama una mayor atención hacia los recursos humanos, la flexibilidad organizativa y la capacidad de innovación. Lo que algunos autores llaman un movimiento de reforma down-grid (DunleaVy y HoOd), neotaylorismo (PoLLITT) o neoweberianismo (RITZER y LeMoyne). Por otro lado, la corrupción no se evita a través de la sacralización del ámbito público, sino a través de un acercamiento al modo de funcionar del sector privado. Lo que, en función de la literatura, se ha denominado reformas down-group (DUNLEAVY y HOOD), agencialización (METCALFE y RICHARDS) o, más directamente, la introducción del espiritu empresarial en el sector público (OsBORNE y GaEBLER).

Considero que la conceptualización de la NGP que ha venido desarrollándose en los fórums académicos y profesionales presenta, como mínimo, dos dificultades. En primer lugar, carece de coherencia interna. Cuando se analizan formulaciones aparentemente coincidentes, aparecen notables diferencias de fondo que dificultan su generalización. Así, después de una breve exploración, todo el mundo parece reconocer que el dinosaurio-APT se ha extinguido, pero a la hora de definir el nuevo dinosaurio-NGP parece que cada autor ha dado con un animal diferente.

La segunda dificultad radica en lo que apuntábamos al iniciar este artículo: la dimensión democrática ha quedado marginada de la mayor parte de las discusiones sobre la NGP. Se detecta una clara obsesión por mejorar la eficiencia de las actuaciones burocráticas, mientras que su democratización o bien se da por supuesta o bien se olvida completamente. Si esto se confirma, estaríamos intentando mejorar la racionalización weberiana -adaptándola a las nuevas tecnologías como sugieren algunos-, pero podríamos estar reproduciendo los problemas de la irracionalidad de la racionalidad. Seguramente sólo se puede formular a modo de interrogante, pero ¿podríamos analizar la guerra del golfo o el genocidio bosnio como la expresión extrema del nuevo proceso de racionalización?

En cualquier caso, el resto del artículo lo dedico a amortiguar - que no a solucionar - las dos dificultades anteriormente mencionadas. Con esta finalidad, distingo tres formas diferentes de observar al dinosaurio-NGP y, al mismo tiempo, intento apuntar las implicaciones democráticas que despierta cada uno de ellos.

\section{La credulidad de la posburocracia}

Entre los teóricos de la NGP encontramos un primer grupo formado por optimistas, entusiastas de los «pos» y, lo que quizá es más peligroso, agoreros capaces de recetarnos soluciones mágicas para los complejos problemas que afectan a nuestras sociedades. Desde su ingeniosa credulidad, articulan un discurso modernizador que les permite esquematizar el paso de la APT a la NGP y, de este modo, visualizar los peldaños que deben subirse para alcanzar un futuro lleno de empleados motivados, líderes carismáticos, estructuras flexibles, presupuestos 
ahorradores y organizaciones orientadas a la satisfacción de sus clientes.

La mayoría de las sugerencias que formulan estos nuevos gurus de la gestión pública se inspiran en el super-ventas de Peters y Waterman, In Search of the Excellence. A partir de la influencia de este libro, la práctica totalidad de las propuestas se articulan alrededor de lo que podríamos considerar las dos máximas de la NGP: gestionar los intangibles y obsesionarse por los clientes.

En primer lugar, se propone gestionar los intangibles porque se considera que el rendimiento de una organización no depende tanto de la gestión de las estructuras y de los procedimientos (los elementos tangibles) como de la gestión de los valores, de las misiones, de los recursos humanos, de los estilos de liderazgo o de las estrategias (los elementos intangibles). Este cambio de énfasis - que Peters y Waterman, utilizando la terminología informática, resumen como el paso del bard al soft- es crucial, ya que rompe con aquella racionalidad weberiana que suponía que la eficiencia se maximizaba institucionalizando -a través de normas y regulaciones- una serie de procedimientos considerados óptimos. Así pues, utilizando un cierto esquematismo, es fácil convertir el paso del bard al soft en el paso de la burocracia a la posburocracia. La lógica es simple pero elegante, aunque -como veremos en el siguiente apartado- esconde algunas falacias.

En segundo lugar, Peters y Waterman han conseguido que la administración pública se llene de clientes, que la mayoría de los responsables de estas administraciones no sepan hablar de otra cosa y que la OCDE haya iniciado una cruzada destinada a recordarnos aquello de "cliente, cliente, cliente ..... Aunque a nosotros el discurso nos parece excesivo, debemos reconocer que la idea es oportuna. La APT se había encerrado sobre sí misma, olvidando que las tareas realizadas no son un fin sino el medio para alcanzar determinados objetivos. Objetivos que, además, tienen que ver con las personas que reciben sus servicios y, aunque esto a menudo se olvida, con la sociedad de la que forman parte. Sea como sea, la obsesión por los clientes también justifica el paso de la burocracia a la posburocracia, ya que representa otra forma de romper con el ideal de la racionalidad weberiana: el individuo no sirve para nada, sólo ha de dejarse llevar por la razón institucionalizada.

Resumiendo, el paso de la burocracia a la posburocracia se concreta en el descubrimiento de los intangibles y de los clientes. Pero ... ¿para qué sirve pasar de la burocracia a la posburocracia? iresuelve este cambio su pérdida de eficiencia? ¿contribuye a paliar su déficit democrático?

Aparentemente, la preocupación por los intangibles facilitaría una mayor eficiencia, mientras que la atención a los clientes podría suponer cierta democratización de las relaciones entre la administración y los administrados. Sin embargo, desde nuestro propio punto de vista, creemos que ambos procesos se dirigen a solucionar problemas de eficiencia, mientras que el déficit democrático continúa -como en el modelo racional weberiano- olvidado. Nos hallamos ante un intento de re-racionalizar una administración que debe hacer frente a un entorno más turbulento, a una sociedad más segmentada, a la introducción de nuevas tecnologías y a unos individuos cada vez más exigentes; pero que en ningún caso se plantea cómo introducir la dimensión democrática en sus formas de actuación. El énfasis en los intangibles es imprescindible para orientar la organización en un mundo que se transforma velozmente, mientras que la preocupación por los clientes ejemplariza la necesidad de dar respuesta a demandas sociales más segmentadas y más individualizadas; pero tanto uno como otro no tienen otra finalidad que recuperar la eficiencia que la racionalidad formal había perdido con el paso del tiempo. Según parece, la democracia es cosa de los políticos, no de los posburócratas.

Vamos a ilustrar las opiniones vertidas en los apartados anteriores a través de una breve referencia a dos autores que, sin menoscabo de sus indudables méritos, consideramos representativos de lo que hemos llamado la credulidad posburocrática. Aunque al incluirlas en este apartado alguien pueda suponer cierto escarnio, queremos subrayar que respetamos tanto la influencia como la capacidad de innovación teórica de estos autores, si bien es cierto que no estamos absolutamente de acuerdo con algunos de sus planteamientos. Estos autores son Osborne-Gaebler y Barzelay.

En primer lugar, el libro Reinventing Government: How the Entrepeneurial Spirit is Transforming the Public Sector de OsborNe y GAEBLER representa, para el sector público, lo que para el privado representó, una década antes, el libro de PETERS y Waterman. Se trata de un super-ventas de gran influencia -sobre todo en el mundo profesional - que ha logrado alterar la forma de observar, de analizar y de hablar respecto a la administración pública. Incluso la estructura del libro es muy parecida a la de su tocayo en el ámbito público: 10 capítulos que, a partir de múltiples ejemplos (a veces anécdotas), presentan los 10 ingredientes básicos para reinventar el gobierno y, de paso, solucionar todos los problemas que aquejan a las administraciones públicas actuales. Ya que OsBorne y GaEBler no están sometidos a las mismas presiones bélicas que el druida Panoramix, no han guardado el secreto de estos 10 ingredientes:

1. Gobierno catalizador: mejor llevar el timón que remar.

2. Gobierno propiedad de la comunidad: mejor facultar que servir directamente.

3. Gobierno competitivo: inyectar competitividad en la prestación de servicios. 
4. Gobierno inspirado en objetivos: la transformación de la organización regida por reglas.

5. Gobierno dirigido a los resultados: financiar el producto, no los datos.

6. Gobierno inspirado en el cliente: satisfacer las necesidades del cliente, no las de la burocracia.

7. El gobierno de corte empresarial: ganar en lugar de gastar.

8. El gobierno previsor: más vale prevenir que curar.

9. El gobierno descentralizado: de la jerarquía a la participación y al trabajo en equipo.

10. El gobierno orientado al mercado: provocar el cambio a través del mercado.

Lo primero que destaca en el libro de Osborne y GaEBler es su ambición: „Mostraremos instantáneas de los gobiernos empresariales ya existentes y esbozaremos diez principios sencillos sobre los cuales parecen haber sido construidos. No ofrecemos estos principios - este 'mapa' - como la última palabra sobre la reinvención del gobierno, sino más bien como un borrador aproximado" (1994: 17). Lo segundo su optimismo: "Nuestro propósito no es criticar al gobierno, sino renovarlo. Tenemos tanta confianza en el futuro del gobierno como desazón ante su situación actual. No ocultamos la magnitud del problema, ni desconocemos la dificultad para resolverlo. Pero, puesto que hemos visto tantas instituciones públicas que, de oscuras burocracias se han transformado en organizaciones innovadoras, flexibles, capaces de reacción, creemos que las soluciones existen de verdadn (1994: 23).

Esta combinación de ambición y optimismo es el resultado de lo que observan como un cambio de paradigma o, utilizando sus términos, una "perestroika norteamericana" que aportará los mecanismos necesarios para superar los límites de la APT e implantar una boyante NGP: “...hay esperanzas. Lenta, silenciosamente, lejos de las candilejas, comienzan a surgir nuevos tipos de instituciones públicas. Son directas, descentralizadas e innovadoras; son flexibles, dúctiles y capaces de aprender con rapidez nuevas formas de acción cuando las condiciones cambian. Emplean la competencia, la elección del cliente y otros mecanismos no burocráticos para que las cosas se hagan lo más creativa y eficazmente posibles. Y son nuestro futuro" (1994: 26).

Así pues, en un futuro próximo, nuestras instituciones públicas pueden llegar a ser fantásticas. OsBorne y GaEBlER, de una forma extraordinariamente sugerente, nos muestran evidencias y nos proponen alternativas de acción. Su discurso no sólo es interesante, sino también convincente. Pero tiene sus límites, ya que - como reconocen los propios autores- no se trata de cambiar lo que hace el gobierno, sino solamente su forma de hacerlo: -Nos preocupamos enormemente por lo que los gobiernos hacen, pero este libro se ocupa de cómo operan. (1994: 22). Reinventar el Gobierno, por lo tanto, puede resultar un título excesivo, pues lo que preocupa a sus autores es como recuperar la eficiencia de las acciones de gobierno, no como mejorar o como -y este es nuestro problema- democratizar sus contenidos. No estamos pues ante un cambio de paradigma gubernamental, sino ante un intento de resolver los límites de la racionalidad burocrática; unos límites que, además, se sitúan exclusivamente en el mundo de la eficiencia. Nunca en el de la democracia.

Quizá el único momento en que podriamos iniciar una discusión sobre la dimensión democrática de las propuestas de OSBORNE y GAEBLER, es cuando plantean la distinción básica entre remary timonear. Afirman que los gobiernos tradicionales se han dedicado básicamente a remar, es decir, han realizado tareas; mientras que los gobiernos reinventados deberían timonear, es decir, dirigir la nave a buen puerto. Llevar el timón significa gobernar y, situados en sistemas democráticos, debería estar conectado con la expresión de la voluntad de los ciudadanos. Son ellos quienes deberían indicar al capitán hacia qué puerto quieren dirigirse, con qué velocidad quieren navegar y siguiendo qué ruta. Unos ciudadanos con estas capacidades recuperarían sus posibilidades de elección y se bumanizarian, lo que constituye el punto de partida para solventar los déficit democráticos de la APT.

Sin embargo, al establecer la conexión entre el timón y la sociedad no aparece la dimensión democrática. El timón no se gobierna a través de la expresión de voluntades colectivas, sino de la introducción de estilos empresariales en el sector público. Después de afirmar en el primer principio la necesidad de timonear, el segundo habla del sector informal, el tercero de la competitividad, el cuarto de la dirección por objetivos, el quinto de los resultados, el sexto de los clientes, el séptimo del talante empresarial, el octavo de la previsión, el noveno de la descentralización y el décimo del mercado. ¿Dónde aparece aquí la democracia? A mi entender, en ninguna parte, ni tan siquiera en la notas a pie de página. La reinvención del gobierno, en definitiva, no aporta ninguna solución para paliar el déficit democrático de la APT. Se concentra, en cambio, en aportar nuevos mecanismos para recuperar la eficiencia que esta APT había perdido. Por un lado, se pierde la oportunidad de bumanizar a los ciudadanos y se opta por un proceso de clientalización de la relaciones entre las administración y los administrados. Esta clientalización puede solventar problemas de eficiencia, pero nunca democratizar a la APT. Por otro lado, la capacidad de elección se encajona en su acepción mercantil. No se trata de escoger entre fines u objetivos, sino entre mecanismos o instrumentos. Tampoco aquí, por lo tanto, encontramos la vía democratizadora que algunos reclamamos. 
Nuestro segundo autor, Barzelay, ha escrito uno de los libros más importantes tanto desde la óptica teórica como desde la empirica: sobre la posburocracia. En Breaking Through Bureaucracy: A New Vision for Managing in Government, BARZELAY define el paso de la burocracia a la posburocracia, al mismo tiempo que ilustra con múltiples referencias prácticas su discurso teórico. La combinación teórico-práctica hace de su obra uno de los libros más consistentes que se han escrito sobre la temática que nos ocupa. En su trabajo se resuelven algunas de las dificultades asociadas al excesivo optimismo respecto a los pos, pero consideramos que ni en su texto ni en sus notas a pie de página se presta la atención necesaria a la dimensión democrática de la NGP.

BaRZELAY se refiere al paso de la burocracia a la posburocracia como un cambio de paradigma, pero no en el sentido khuniano de revolución (el utilizado por OSBORNE y GAEBLER) sino en un sentido más suave de evolución. Ya se intuye, por lo tanto, que no está refiriéndose a algo sustancialmente diferente, sino a una especie de mutación adaptativa desde un paradigma originario a otro derivado. BARZELAY es muy claro al respecto: sugerimos que el nuevo paradigma se entiende mejor a partir de la metáfora de una familia extensa de ideas. La imagen de una familia extensa es de gran utilidad, ya que nos recuerda que cada idea se relaciona con las otras y, por lo tanto, se requiere cierta reflexión para detectar estas relaciones (1992: 116).

A partir de estas ideas, Barzelay define esquemáticamente los rasgos básicos de cada paradigma:

\begin{tabular}{|c|c|}
\hline 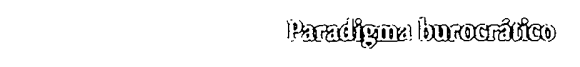 & 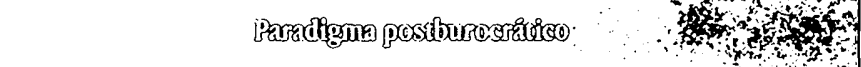 \\
\hline $\begin{array}{l}\text { - interés público } \\
\text { - eficiencia } \\
\text { - administración } \\
\text { - control } \\
\text { - funciones, autoridad y estructuras especificadas } \\
\text { - justificación de costes } \\
\text { - responsasbilidad impuesta } \\
\text { - reglas y procedimientos } \\
\text { - sistemas administrativos }\end{array}$ & $\begin{array}{l}\text { - valor de los resultados para los ciudadanos } \\
\text { - calidad y valor } \\
\text { - producción } \\
\text { - adhesión a las normas } \\
\text { - identificar misiones, servicios y resultados } \\
\text { - valor de la prestación } \\
\text { - construir responsabilidad y atender recursos humanos } \\
\text { - entender las normas, comprometerse con los problemas e innovar } \\
\text { - introducir el mercado, la elección, los valores, los incentivos, la evalua- } \\
\text { ción y la acción colectiva }\end{array}$ \\
\hline
\end{tabular}

Fuente: adaptado de Bar7FIAY (1992: 118).

Después de analizar atentamente el cuadro anterior y de observar como el propio Barzelay explica cada uno de sus items, opino que la posburocracia sólo podría provocar una democratización de la administración pública si las preocupaciones por los valores y por los ciudadanos se conectaran a través de unos mecanismos electorales revitalizados. Es decir, si las misiones o los objetivos se articularan a partir de la participación efectiva del conjunto de los ciudadanos, si se reconociera que los valores que inspiran las organizaciones públicas representan voluntades colectivas y no únicamente estrategias de adaptación al entorno. Esta conexión entre valores y opciones ciudadanas reclama la presencia de individuos bumanizados y, por lo tanto, podría afrontar los déficit democráticos de la APT.

Sin embargo, se prefiere establecer la conexión a través de la responsabilidad (accountability). Una responsabilidad que se interpreta como la capacidad de respuesta ante las demandas de los clientes (responsiveness), mientras que deja en segundo plano la construcción de opciones colectivas (governance). Es decir, se trata de una responsabilidad frente a la eficiencia, no frente a la democracia. Si recordamos a WEBER, podemos concluir que estamos hablando de neoburocracia, no de posburocracia. Y esta neoburocracia, insistimos, no resuelve los déficit democráticos de la burocracia.

\section{La desconfianza del neoweberianismo}

El propio Hood describe la extinción del dinosaurio-APT con cautela. Recoge las fuertes transformaciones que está experimentando la administración pública actual, pero reconoce que puede tratarse más de una adaptación (de segunda o tercera generación) de la burocracia weberiana a los cambios sociales y tecnológicos que de una transformación radical: aA menudo ... la NGP parece separarse efectivamente de la modernización administrativa weberiana. Pero, otras veces, se nos muestra como un paso más en el desarrollo de la burocracia weberiana" (1994: 138).

Esta argumentación es explorada con mayor profundidad por HECKSCHER. En su trabajo Defining the Post-Bureaucratic Type, este autor nos advierte de lo que considera las falsas definicio- 
nes de la posburocracia. En su opinión, estas falsas versiones no son otra cosa que formulaciones neoburocráticas, aunque a menudo la novedad de los mecanismos de actuación provoque su clasificación dentro del atractivo mundo de los pos. Concretamente, HECKSCHER se refiere a tres falsos modelos posburocráticos o, en otras palabras, a tres variantes neoburocráticas: la burocracia mejorada, la alternativa comunitaria y el modelo mercantil.

La primera variante es la más frecuente, pues la mayoría de las iniciativas modernizadoras se dirigen a la mejora y a la renovación de la burocracia, no a su transformación. A menudo se ha confundido la posburocracia con estas iniciativas, pero su divergencia es clara. Por ejemplo, uno de los postulados más corrientes entre la falsa posburocracia sostiene que las organizaciones públicas deben descentralizarse, gestionar a través de objetivos y potenciar la autonomía de sus órganos. El lenguaje de la descentralización, de los objetivos y de la autonomía puede parecer novedoso, pero no es más que un redescubrimiento de las raíces del modelo weberiano: la jerarquía y la especialización. Lo mismo sucede con los falsos posburócratas de los circulos de calidad: sugieren confiar en los trabajadores y proporcionarles una mayor autonomía de acción, pero esto no se refiere a sustituir la burocrácia sino a estimular su buen funcionamiento. DRUCKER, por poner otro ejemplo popular, se refiere a la emergencia del modelo posburocrático a través de una metáfora: el paso de una miríada de canta-autores o de las pequeñas bandas musicales a la orquesta sinfónica, donde se amalgaman múltiples esfuerzos para conseguir un único objetivo. Obviamente, la imagen de un director controlando a decenas de músicos puede ser. muy sugerente, pero es una imagen básicamente ligada al modelo burocrático. En general, por lo tanto, nos encontramos con una serie de iniciativas que -a través de la autonomía, de la descentralización y de la definición de objetivos- pretenden situarnos en un escenario posburocrático, cuando lo único que hacen es preocuparse por el buen funcionamiento de la burocracia. Lo que, en cualquier caso, no es poco!! OsboRne y GaEBler, y este es nuestro último ejemplo, utilizan una cita del general Patton para ejemplarizar el espíritu del nuevo paradigma: "Nunca digas a la gente cómo tiene que hacer las cosas. Di qué quieres lograr y te sorprenderá con su ingenio". Este responsabilizar a cada uno de sus actos no es más, según HECKSHER, que un claro instinto burocrático.

La segunda falsa variante posburocrática es la alternativa comunitaria. Autores como SCHEIN, vinculan el afianzamiento del nuevo paradigma al desarrollo de culturas organizativas fuertes y unificadas. A través de ejemplos como IBM, Microsoft o, más genéricamente, de referencias al milagro janonés, argumentan que una organización que comparte valores puede favorecer la unidad de objetivos y, al mismo tiempo, facilitar un control sin reglas; es decir, un control no burocrático sobre la organización. La alternativa comunitaria introduce algún elemento posburocrático: la confianza, la gestión de la irracionalidad o la organización informal. Pero todavía presenta algunos problemas típicos de las organizaciones burocráticas. El modelo comunitario incorpora elementos corporativos y de solidaridad grupal que pueden limitar la flexibilidad y la adaptación a las variaciones del entorno. La rigidez y la incapacidad de relación con el exterior son problemas eminentemente burocráti$\cos$, aunque ésta haya mejorado su funcionamiento a través de cierto cambio en las actitudes de los empleados.

Finalmente, el denominado modelo mercantil se presenta a menudo como una variante posburocrática. Muy esquemáticamente, este modelo postula la aplicación de criterios mercantiles a las organizaciones públicas, siempre bajo el supuesto de que los mercados son más eficientes que las jerarquías burocráticas. Se trata de mejorar las relaciones interorganizativas a través de vínculos contractuales, aunque se suele olvidar que -especialmente cuando se trata de alcanzar objetivos colectivos- el contrato no es el mecanismo más adecuado para fomentar la cooperación. Las relaciones contractuales pueden incluso provocar resultados paradójicos: la fijación de los objetivos se convierte en algo oscuro y distante, los miembros de la organización son incapaces de entenderla de forma global y, consecuentemente, cada uno de ellos se concentra en sus propias actividades, compitiendo con los demás y deteriorando cualquier posibilidad de diálogo interorganizativo. El modelo mercantil, otra vez, se construye sobre un ingrediente básico de la burocracia: la separación de los miembros de la organización en departamentos estancos que sólo se integran en la cúpula de la jerarquía.

A partir de reconocer las falacias anteriormente apuntadas, los teóricos de la NGP han reaccionado de dos formas. Algunos intentan afinar el discurso y, así, construir un modelo posburocrático que efectivamente no sea una revisión sino una transformación de la APT. Otros, en cambio, simplemente consideran que la NGP supone una re-racionalización de la burocracia weberiana y que como tal hay que estudiarla. Me referiré a las aportaciones del propio HeCKSCHER para ilustrar la primera posición, mientras que utilizaré las propuestas de RITZER para la segunda. Para cada uno de ellos resumiremos sus propuestas y discutiremos la ubicación que le dan a la dimensión democrática.

HECKSCHER, en primer lugar, considera que para plantear la existencia efectiva de una administración pública posburocrática es necesaria una redefinición previa de los pilares sobre los que se asienta la burocracia. De otro modo, lo que hacemos no es más que una revisión de la propia burocracia. De este modo, HeCKSCHER inicia su propuesta identificando las debilidades básicas de la burocracia; es decir, no aquellas relacionadas con una implementación deficiente de sus principios, sino aquellas 
que son inherentes a su propia naturaleza. Estos límites de carácter fundamental se concretan en su apuesta por la especialización o, utilizando otros términos, en su preferencia porque cada persona sea responsable únicamente de la tarea específica que realiza. Esta segmentación de responsabilidades propicia la eficiencia burocrática, pero también introduce algunas consecuencias no deseadas: la pérdidad de capacidades potenciales de sus miembros, la dificultad para controlar los elementos informales de la organización, o la falta de flexibilidad que limita las posibilidades de cambio y de adaptación.

A partir de lo expuesto hasta el momento, HECKSCHER - en la línea de otros autores como KoOIman y Dunsire- define la posburocracia como un modelo organizativo donde cada individuo es responsable frente al éxito conjunto de la organización. Este planteamiento sí que nos separa de la burocracia, aparte de permitirnos mejorar algunas de sus debilidades. La posburocracia, por lo tanto, sustituiría la segmentación por la comunicación, la verticalidad por la coordinación, la unidireccionalidad por la bidireccionalidad y la autoridad por la interacción. En palabras del propio Heckscher: aTodo ello nos sugiere un término más positivo para reemplazar la tan llevada y traída posburocracia: a raíz del crucial papel de un diálogo interactivo que vendría a sustituir las órdenes o las comunicaciones unidireccionales, le llamaré 'modelo interactivo' (1994: 24).

La principal dificultad a la hora de articular este nuevo tipo interactivo, tal como reconoce el propio autor, radica en la definición del papel que ha de jugar el poder y la autoridad; lo que nos traslada a la dimensión político-democrática de la NGP. Del modelo posburocrático propuesto por HeCKSCHER se deduce una reducción o, cómo mínimo, una transformación en el uso del poder. De ahí la importancia de plantearse cómo una organización de este tipo puede garantizar que impulsará decisiones investidas de autoridad. A este interrogante se responde afirmando que se puede decidir con connotaciones de autoridad o de obligatoriedad sin depender de una jerarquía segmentada, siempre y cuando seamos capaces de establecer consensos legitimadores. Este consenso legitimador no puede obtenerse a través de otra vía que no sea la democratización de las administraciones públicas. Una democratización que permite llegar a acuerdos, al mismo tiempo que proporciona a la organización los principios y los valores que han de inspirar su proceso de decisión. Utilizando las palabras del mismo autor: -La integración del orden posburocrático no requiere un proceso de creación de consenso para cada decisión, sino que cada decisión se tome de acuerdo con unos principios que han sido desarrollados en dicho proceso" (1994: 41).

Así pues, a diferencia de lo que apuntábamos en el apartado anterior, la propuesta posburocrática de HECKSCHER recupera la dimensión democrática de la administració pública. El nuevo modelo no está compuesto por personas deshumanizadas y sin capacidad de elección, sino por individuos que deben colaborar, participar, negociar, alcanzar acuerdos y valorar sus actuaciones en función de los objetivos de la organización. Sin entrar ahora en las dificultades de articular una organización como la descrita, lo cierto es que su potencialidad va más allá de la simple resolución de los problemas de eficiencia de la burocracia y se sitúa en el ruedo de los déficit democráticos. Una organización que recupera sus personas y sus valores es una organización que, como mínimo, aborda la dimensión democrática de la administración pública.

Rutzer, nuestro segundo ejemplo de desconfianza, es todavía más desconfiado. HeCKSCHER desconfiaba de la mayoría de los pos, pero acaba aportando su propia solución. RuTzer, en cambio, no sólo desconfía de los pos propuestos por otros autores, sino que no cree en la posibilidad de construirlos. A diferencia de HECKSCHER, RITZER cree que no nos estamos dirigiendo hacia la posburocracia sino hacia una re-racionalización de la tradicional burocracia weberiana. En este sentido, Ritzer es el mejor exponente de lo que aquí he denominado neoburocracia.

En su último trabajo, The McDonaldization of Society, RITZER analiza cómo la sociedad está evolucionando desde la modernidad weberiana hacia una modernidad profundizada a través de lo que él bautiza como el proceso de McDonaldización. El progresivo asentamiento de la McDonaldización no supone el descubrimiento de ningún nuevo estadio social, sino que se asienta sobre las premisas básicas de la racionalidad weberiana. El triunfo del proceso de McDonaldización se construye, precisamente, sobre su capacidad para ofrecer a los empleados, a los directivos y a los propios consumidores un marco de eficiencia, calculabilidad, predictabilidad y control tecnológico. Los mismos rasgos que, como hemos visto anteriormente, definían la racionalidad formal de WEBER.

RUTZER expresa su posición con rotundidad: "Así pues, los McDonalds y, más genéricamente, el proceso de McDonaldización no representa nada nuevo, sino más bien la culminación de una serie de procesos de racionalización que se han ido sucediendo a lo largo del siglo $\mathrm{XX}^{n}$ (1996: 31). Y esta posición tiene importantes efectos sobre el debate entre la APT y la NGP. El proceso de McDonaldización aplicado al ámbito de la administración pública no rompe las premisas burocráticas sino que reproduce sus mecanismos de control centralizado, su jerarquía interna, su tendencia a la especialización y la mecanización de las tareas, o su producción rutinizada de objetos uniformes y estandarizados. Aunque, quizá, los reproduzca con mayor profundidad debido a la disponibilidad de nuevas y más sofisticadas tecnologías. Es decir, no se detecta ningún movimiento de reforma hacia la NGP, sino que se reafirma, aunque con algunas modificaciones técnicas, la APT. Simplemente para ejemplarizar estas afirmaciones podríamos referirnos a las pala- 
bras de Ray Kroc, el empresario que compró el primer McDonald a los hermanos que lo habían fundado y que lo convirtió en lo que actualmente es: -Quedé fascinado por la simplicidad y la efectividad del sistema (...), cada paso en la producción de un menú limitado se desmenuzaba hasta su esencia y se realizaba con un mínimo esfuerzo. Sólo vendían hamburguesas y (...) cada hamburgesa era cocinada exactamente de la misma manera. (citado en RITZER, 1996: 37).

El proceso de McDonaldización, por lo tanto, ofrece los mismos beneficios que había representado la racionalidad burocrática; aunque, también como su predecesora, sufre de lo que anteriormente hemos denominado sla irracionalidad de la racionalidad. La McDonaldización genera procesos de deshumanización y limita la capacidad de elección de los individuos (muy claros en el trato con sus empleados y sus consumidores) que, en definitiva, reproducen el déficit democrático que ya sufría al modelo weberiano.

Se detectan irracionalidades que producen ineficiencia, despilfarro o ineficacia, mientras que otras afectan a la dimensión democrática del modelo. Entre las primeras destacan los efectos no deseados producidos por una mano de obra desmotivada y en rotación permanente, por la pérdida de iniciativa, por el encarecimiento de unos productos de muy baja calidad, o por la imposibilidad de adaptarse con rapidez a los cambios. Es decir, los mismos problemas que sufría el modelo burocrático clásico. El propio RITZER nos propone un ilustrativo ejemplo de la irracionalidad de la racionalidad aplicado al mundo de la aviación. Los Boeing 757 y 767 son los más modernos y computarizados aviones del mercado. En lugar de ser pilotados personalmente o de utilizar los viejos pilotos automáticos (que únicamente servían para realizar las maniobras más simples), estos aparatos pueden despegar de un punto, volar y aterrizar en otro lugar sin que el piloto haya hecho otra cosa que apretar una serie de botones en su computadora. El control humano se reduce al mínimo, mientras que la tecnología se hace responsable de la mayor parte del trabajo. Estos aviones pueden ser más seguros que los anteriores, ya que eliminan cualquier posibilidad de error humano. Sin embargo, una generación de pilotos acostumbrados a depender de estas tecnologías puede convertirse en una generación de pilotos sin experiencia de vuelo, sin capacidad de reaccionar ante situaciones delicadas, o sin creatividad para resolver emergencias. La deshumanización del trabajo de los pilotos puede, en estos casos, llegar a ser irracional y producir la muerte de los pasajeros por la falta de habilidad y de experiencia del factor humano.

Centrándonos ya en los problemas democráticos, RirzFr cree que el proceso McDonaldización nos conduce a la misma ijaula de acero de la que nos había advertido el propio WfBER. Esta ajaulla de acero" se manifiesta en la difusión de la McDonaldización hacia todos los aspectos de nuestras vidas y hacia todos los rincones del mundo; una difusión que nos atrapa y nos impide es- caparnos del deshumanizador proceso de racionalización. En esta jaula de acero los individuos pierden toda posibilidad de control tanto sobre sus vidas como sobre el sistema social donde éstas se desarrollan. Como ya hemos repetido insistentemente, un mundo formado por individuos sin capacidad de elección y sin posibilidades de ejercer ningún control sobre su entorno es un mundo profundamente anti-democrático.

Hemos utilizado los argumentos de Rutzer para presentar un caso donde se observa el paso de la APT a la NGP como una falacia, pues se define el tránsito del weberianismo a la McDonalización como un movimiento desde la APT hasta másAPT. Sin embargo, el libro de RITzER va más allá de esta afirmación. Este autor no sólo nos propone una crítica a la posburocracia, sino a todos los pos (posfordismo, posmodernismo, posindustrialismo) que afectan a las sociedades actuales: “Dado que he presentado la McDonaldización como un proceso central en el mundo 'moderno', este libro constituye un análisis y una crítica de la 'modernidad'. Sin embargo, desde un buen número de perspectivas contemporáneas -en especial el posindustrialismo, el posfordismo y el posmodernismo- se afirma que ya hemos superado el mundo moderno y que nos encontramos ante una sociedad radicalmente nueva. Estas posturas implican que éste es un libro retrógrado, pues se ocupa de un fenómeno 'moderno' (la McDonaldización) que pronto desaparecerá. Defiendo, sin embargo, que la McDonaldización y sus rasgos 'modernos' (también industrialistas y fordistas) no sólo estarán presentes en el futuro, sino también que su influencia social tiende a incrementarse" (1996: 148).

Sin tomar partido por la modernidad o por la posmodernidad, lo cierto es que el libro de RITzER presenta argumentos claros y contundentes que avalan sus posiciones más desconfiadas. En este sentido, representan un soplo de aire fresco respecto a la avalancha de pos que nos ha sacudido en los últimos años y que nos ha hecho creer que en cada esquina podíamos encontrar una nueva forma de interpretar el mundo. Pero la desconfianza de Ritzer no es únicamente un antídoto a nuestros entusiasmos teóricos, sino que también nos aporta una buena dosis de realismo empírico. Explicar el proceso de McDonaldización en los términos de RITZER significa aterrizar en la realidad. En la realidad de los efectos más indeseados de la robótica, del trabajo precario, de la deshumanización de las relaciones entre los individuos, de los consumos teledirigidos, del vacio de las populares generaciones $X$. Una realidad que únicamente podemos reconocer sin entusiasmo y que, ya para finalizar este apartado, repercute negativamente en la democratización de la administración pública y, más genéricamente, del conjunto social. La conclusión es pesimista, pues podríamos afirmar que la McDonaldización no sólo reproduce los déficit democráticos del modelo weberiano, sino que los intensifica. 


\section{La crítica desde la especificidad del ámbito público}

La fuerza de los argumentos posburocráticos, ejemplarizada en la popularidad de autores como Osborne y GaEBler o Peters y WATERMAN, ha situado en la marginalidad las críticas a la NGP. A veces se considera que estas críticas suponen un atrincheramiento en las premisas más recalcitrantes de la burocracia weberiana o en la defensa a ultranza de un Estado de bienestar difícil de mantener; pero no se trata únicamente de ésto. Las posturas críticas pueden reconocer la necesidad de cambio y las virtudes de la NGP, pero no por ello dejar de reconocer sus límites o sus contradicciones.

Quizá uno de los primeros autores en apuntar de forma sistemática las dificultades de la NGP sea PoluTT. En su trabajo, Managerialism and the Public Services, este autor senala cuatro posibles críticas a lo que él considera un desarrollo excesivamente neotaylorista de la NGP: críticas a su coherencia, a su realismo, a sus intereses y a sus valores.

En primer lugar, detecta tensiones y contradicciones que dificultan la coherencia interna del modelo. Se trata de incoherencias que aparecen cuando nos alejamos de las grandes declaraciones de intenciones y nos acercamos a temas más operativos como la motivación de los empleados públicos, la participación democrática, o los procesos simultáneos de delegación-control.

En segundo lugar, Pouutr califica los postulados neotayloristas como poco realistas, pues considera que asumen la posibilidad de trasladar la gestión privada al ámbito público sin darse cuenta de las especificidades de éste último. Concretamente, alude a rasgos distintivos como la responsabilidad de los representantes electos, la diversidad y el antagonismo de sus objetivos, la usual ausencia de competencia, la inexistencia de una relación clara entre el volumen de servicios ofertados y los recursos obtenidos, o la presencia de una ciudadanía con unas connotaciones colectivas distintas del individualismo de los clientes.

En tercer lugar, PolutT nos recuerda que el programa neotaylorista no es un mero ejercicio de ingeniería técnica, sino que articula un elenco de intereses muy determinados. La lista de beneficiarios de la NGP es muy ilustrativa: empresas auditoras, escuelas de negocios, empresas interesadas en la subcontratación de servicios públicos, políticos neoliberales, etc.

Finalmente, PoluTt también nos advierte de la dimensión valorativa del neotaylorismo. Su compromiso con la economía y la eficiencia (siendo la eficacia una especie de hermano pobre) deja de lado otros valores como la igualdad, la justicia, la representación o la participación. Se trata, en opinión del autor, de una firme apuesta por el individualismo. De una apuesta que no es neutral y que, consecuentemente, afecta tanto a lo qué hace como a la forma de actuar de la administración pública.

En el marco del debate sobre los valores que incorpora la NGP, DuNLEAVY y Hood dan un paso de vital importancia, ya que lo sitúan en un eje izquierda-derecha de gran utilidad para dotarlo de contenido ideológico. De este modo, a partir de las teorías culturales de SCHWARZ y THOMPSON, estos autores utilizan distintas valoraciones sobre la NGP para dibujar opciones y escenarios de futuro que se nos presentan en clave político-ideológica.

En primer lugar, los convencidos de la bondad del mercado nos proponen lo que DunlEavy y Hood llaman la acrítica individualista. Desde ella se afirma que la NGP corre el riesgo de convertirse en un sustituto mediocre de los derechos contractuales individuales. Lo más recomendable, por lo tanto, es potenciar la privatización y la desregulación. De forma muy esquemática, los individualistas - situados obviamente en la derecha del espectro político- proponen más contratos y menos cuasi-contratos, más mercado y menos cuasi-mercados. Se trata de una opción que diluye a la administración pública y pone en duda su propia existencia.

En segundo lugar, desde la denominada "crítica jerárquica" se sugiere que la NGP podría erosionar la cohesión del sistema administrativo y, de este modo, situar al sector público fuera de control. Es decir, la NGP puede provocar la pérdida de dirección global, de diseño estratégico y, así, erosionar la ética del servicio público y desestabilizar su proyecto de futuro. La alternativa propuesta por los jerárquicos pasaría por fortalecer la capacidad de control central e incrementar las obligaciones de consulta. Se trataría, en términos más operativos, de potenciar los órganos reguladores, de mejorar las relaciones contractuales, de establecer mecanismos de influencia efectiva y, utilizando un lenguaje más teórico, de sustituir las llamadas relaciones contractuales de delegación por unas nuevas relaciones de obligación. En otras palabras, se trata de gestionar con convicción las relaciones en un mapa administrativo cada vez más fragmentado, ya que de otra forma no se puede controlar ni dirigir. Y sin control ni dirección no hay gobierno. La *crítica jerárqui$c a^{\nu}$, en definitiva, reivindica un ejercicio efectivo de la democracia representativa, que se traduce en fomentar el papel de unos políticos que han de marcar las líneas estratégicas de las acciones de la administración pública. Nos situamos pues más allá de la regulación mercantil y, por lo tanto, nos apartamos de las opciones más claramente de derechas. Concretamente, dentro del mapa político actual, parece que los partidos de centro son los más cercanos a estas posturas.

Finalmente, las opciones ubicadas más a la izquierda del espacio político parecen alinearse con una tercera postura: la "crítica igualitaria. Para los igualitaristas, la dimensión 
fragmentadora y empresarial de la NGP puede convertirse en una fuente de corrupción, de defensa de los intereses de algunos altos cargos, y de responsabilidades debilitadas. La alternativa propuesta pasaria por ceder más poder a los ciudadanos, convertidos en garantes del control y la transparencia. Se trata, por lo tanto, de una alternativa que reivindica una democracia más participativa, más descentralizada y más pendiente de la ciudadanía; un concepto que -como ya he señalado- a menudo parece olvidar la NGP.

Personalmente, considero que mientras la primera crítica nos sitúa en un escenario claramente de derechas, donde el individualismo y el mercado se introducen como la única alternativa para mejorar la administración pública; la segunda y la tercera pueden ser complementarias y configurar un escenario tendencialmente de izquierdas, donde se combinan las propuestas de liderazgo público y de participación ciudadana. Centrándonos en la dimensión democrática de la NGP, no me parece disparatado argumentar que el primer escenario la deja de lado, mientras que el segundo la sitúa en el centro de su discurso. Desde la crítica individualista no es necesario buscar mecanismos democráticos para resolver los conflictos que aparecen en la administración, pues parece que estos conflictos o bien son técnicos o bien se han de resolver a través de la autorregulación mercantil. Para los jerárquicos y los igualitaristas, en cambio, la administración pública debe fijarse unos objetivos políticos y, si pretende ser democrática, hacerlo a través de la participación de los ciudadanos.

A partir de este punto, por lo tanto, podemos plantear una auténtica alternativa democrática a los planteamientos más radicales de una NGP que, bajo la tutela de las modas neoliberales, ha acentuado sus aspectos estrictamente neotayloristas. Pero, ¿cómo se articula está alternativa? ¿cómo podemos operativizar un discurso que hasta el momento hemos formulado en clave más crítica que constructiva?

No soy tan presuntuoso como para ofrecer mi respuesta a estos interrogantes, sino que - tal como he hecho hasta ahora- prefiero referirme a algunas aportaciones recientes que sin agotar el debate pueden ofrecer algunos elementos de reflexión. En esta dirección me ha parecido de enorme interés el trabajo de RANSON Y STEWART, promotores de una redefinición de la NGP que en el mundo académico se conoce bajo el acrónimo PSO (public service orientation).

En el libro Management for the Public Domain, Ranson y STEWART reconocen que las críticas al despilfarro, a la ineficiencia y a la ineficacia de la APT son acertadas; pero subrayan que una alternativa como la de NGP debe referirse a alguna cosa más que a la disfuncionalidad interna de estas administraciones. Explícitamente, por lo tanto, evitan centrarse en la dimensión operativa y, como veremos enseguida, se sitúan en una óptica político-democrática muy útil para responder a los interrogantes planteados anteriormente.
RAisOX y STEWaRT representan las posturas más críticas dentro del mundo de los estudiosos de la gestión pública, ya que para ellos el discurso instrumental es secundario $y$, en cambio, proponen recuperar la esencia políica de la NGP. Para estos autores, la gestión pública se caracteriza por la universalidad de sus objetivos, por expresar valores de índole política, por dedicarse a reconciliar la diversidad de intereses sociales y por mediar entre los intereses particulares y el interés colectivo. De este modo, creen que cualquier propuesta para diseñar una reforma administrativa —objeto de la NGP — no puede quedar agotada en sus aspectos instrumentales, sino que debe fomentar la aparición de una nueva moral y de un nuevo orden politico que responda a las necesidades de una sociedad en profunda transformación.

La administración pública expresa valores y, en este sentido, Ranson y Stewart distinguen entre el dominio de los valores socialdemócratas que caracterizó a la APT desde los años cincuenta y el dominio de los valores mercantiles que define las más populares corrientes de la NGP. Durante la etapa socialdemócrata, el profesionalismo -que se manifestaba a través de una burocracia racional, pragmática y tecnocrática- proporcionaba a la administración pública un marco donde hacer operativos sus objetivos de igualdad y universalidad. Con la reciente aparición del modelo consumerista —concepto con el que se refiere a la NGP - los antiguos objetivos de igualdad y universalidad han dejado paso a los de la triple-E (economía, eficiencia y eficacia). La persecución de estos objetivos, además, ya no se realiza desde el profesionalismo sino desde la introducción de mecanismos de mercado en la administración pública.

Sin embargo, para RANSON Y STEWART, el modelo consumerista presenta un problema básico: ¿cómo dar forma a la responsabilidad y a la legitimidad pública que deben acompañar a cualquier acto de la administración pública? Creen que cualquier intento de modernizar la administración pública ha de hacer frente a un dilema de difícil solución: lograr con economía, eficiencia y eficacia la obtención de unos resultados capaces de reconciliar los intereses individuales y los colectivos. Se trata de combinar la dimensión instrumental con la dimensión política. Pero esta combinación no se obtiene a través del mercado, ya que, por definición, el mercado no deja espacio para la lógica política.

Para explicar con más precisión los problemas de un modelo consumerista excesivamente dependiente del mercado, Ranson y STELART utilizan los conceptos exil y vovice elaborados por Hirschman hace ya más de dos décadas. Para este autor, los sistemas complejos se regulan a través de dos mecanismos alternativos: la posibilidad de optar por una alternativa (exit) y la posibilidad de expresar opiniones (voice). En el mercado los individuos pueden optar por otro producto u otra empresa (exit), 
y es precisamente esta posibilidad la que regula su funcionamiento. En el sector público, en cambio, la opción exit es muy limitada y, por lo tanto, su funcionamiento depende de la opción voice, es decir, del derecho de los ciudadanos a expresarse y a ser escuchados. Evidentemente, si incorporamos la opción voice a la NGP estamos adentrándonos en la posibilidad de solventar los déficit democráticos de la APT. En la APT no existía la opción exit; pero tampoco la voice, ya que la única voz que se debía escuchar era la de la racionalidad y la técnica. Una NGP democratizada, por lo tanto, es aquella que articula el derecho de los ciudadanos a expresarse y a ser escuchados. Aquella que, además, es capaz de transformar las presiones y las protestas de los ciudadanos o de grupos de ellos en discusiones democráticas que sirvan para legitimar las actuaciones de la administración pública.

¿Cómo se consigue esta NGP democratizada? En primer lugar, reconociendo que la gestión pública sirve para dar expresión a una gran diversidad de objetivos en competencia y que, consecuentemente, se trata de una actividad política. Esta afirmación no sólo es extraña para muchos de los defensores de la NGP, sino que incluso suscita sus más convencidos ataques. Tal como observa PolutT: "Si alguien pregunta 'cómo' vamos a conseguirlo, la respuesta de los partidarios del modelo gerencialista es muy contundente: a través de la introducción de unas prácticas de gestión adecuadas, las cuales se asume que se encuentran en el sector privado. Tan importante como lo anterior, consideran que la política no puede aportar ninguna mejora, pues se trata de una actividad observada, en el mejor de los casos, como ineficiente y seccional y, en el peor, como irrelevante y conflictiva" (1993: 7). Este tipo de afirmaciones son precisamente las que combaten autores como Ranson y STEWWART o el propio PolulTt. Muy al contrario, consideran que la gestión pública ha de ser la expresión de un proceso político.

En segundo lugar, el carácter político de la administración pública se concreta en la recuperación de un concepto de ciudadania que permita la expresión activa de las opiniones los individuos (voice) y que, por lo tanto, se aparte de la pasividad que se deduce de la clientalización que propone el modelo consumerista. Esta expresión de la ciudadanía ha de ser utilizada para generar, a través de un discurso abierto y pluralista, la legitimidad política de la administración pública.

Así pues, la elección colectiva que legitima democráticamente la actuación de la administración ha de ser articulada a través de un discurso político. Esta elección se determinará a través de las instituciones públicas o, mejor todavía, de la votación de los ciudadanos. De forma parecida a HeCKSCHER, RanSON y StEwarT creen que la elección colectiva depende de la capacidad de alcanzar consensos en relación a los discursos públicos. Consensos que en el caso de sistemas políticos democráticos no se pueden lograr sin la participación activa de los ciudadanos.
Expresar y dar concreción a las voces y a las opiniones de la ciudadanía es pues la responsabilidad última de los gestores públicos. No se trata, como en las propuestas de BarzelAY, de una responsabilidad circunscrita a la capacidad de respuesta frente a demandas de los clientes, sino de una responsabilidad política que se ejerce ante el conjunto de la comunidad. En palabras de RANSON y STEWART: aLa apertura a los ciudadanos es la condición principal que debe satisfacerse para conseguir la responsabilidad y el consenso característicos del ámbito público. Esto presupone una extensión del proceso político, así como el desarrollo de la participación y del discurso político en el marco de un sistema de democracia representativa. (1994: 94).

En resumen, RANSON y STEWART nos proponen una NGP que reconozca y exprese su dimensión política, que sirva para articular discursos colectivos y que sea capaz de escuchar a la ciudadanía. El déficit democrático de la burocracia weberiana es pues la auténtica preocupación de estos autores. Una preocupación que les conduce a reivindicar la bumanidad y la capacidad de elección de unos individuos que han de explicar a la administración pública qué es lo que, colectivamente, esperan de ella. En la última parte de su libro nos hacen algunas sugerencias sobre como alcanzar este objetivo, pero éste ya es un asunto que desborda el objetivo de nuestro artículo.

\section{Conclusiones}

Antes de redactar estas conclusiones he repasado lo que había escrito hasta el momento, intentando decidir si atreverme a formular alguna idea personal, si limitarme a resumir el esqueleto de lo ya expuesto, o si utilizar este final para abrir unos interrogantes de futuro que pudieran garantizar que se me volverá a contactar para escribir un nuevo artículo. Cualquier elección es digna, pero me han parecido poco adecuadas para una aportación que no pretende otra cosa que resumir una serie de aportaciones académicas, así como suscitar un cierto debate en torno a ellas. Mis valoraciones ya han sido expuestas, un breve resumen es innecesario cuando no se pueden enumerar las conclusiones y, finalmente, una agenda de futuro puede acabar por difuminar los auténticos objetivos de este trabajo. Quizá pueda, sin embargo, mezclar estos tres tipos de conclusiones a partir de un par de interrogantes: ¿de qué hemos hablado? y ¿de qué no hemos hablado?

¿De qué hemos hablado? El título del artículo pretende resumir con precisión su objeto; es decir, hablar sobre la dimensión democrática de la nueva gestión pública. Se trata de un objetivo fácil de formular, pero difícil de operativizar. Tradicionalmente, la administración pública ha representado el mundo de la técnica, del profesionalismo, de la ejecución y de una neutralidad que, si me apuran, poco tiene que ver con los dis- 
cursos democráticos. Más recientemente, en cambio, se viene reconociendo que la separación política-administración es una ficción analítica. Pero no se va más allá: después de aceptar que el espacio administrativo es un espacio de conflicto, de relaciones de poder y de elección entre opciones alternativas; parece que hayamos quedado agotados y que no seamos capaces de llevar nuestros argumentos hasta el final. Resulta incoherente afirmar con convicción que la dicotomía wilsoniana pertenece al pasado y, acto seguido, proponer un programa de modernización administrativa que parte de las mismas premisas. Creo que ésta es la incoherencia que hemos experimentado la mayor parte de las personas interesadas en el análisis o en la mejora de la administración pública.

Explicar esta incoherencia y reivindicar la necesidad de superarla podría ser el fin último de este artículo. Para ello he repasado algunos de los autores más significativos de la literatura actual, observando como -salvo meritorias excepcionessus discursos no consiguen superar el esquematismo de la separación decisión-ejecución. Incorporar el poder, el conflicto o las opciones en el estudio de la administración pública no supone simplemente aceptar su existencia, sino que reclama su inclusión en las propias estructuras administrativas. Además, en el marco de un sistema político democrático, esta inclusión debe hacerse a partir de criterios democráticos, ya que, de otro modo, convierte los conflictos y la luchas de poder en una pelea donde las fuerzas son desiguales y donde, por lo tanto, tienden a ganar siempre los mismos.

En resumen, la pauta argumental tiene que ser muy clara: si la administración pública no es un mero instrumento ejecutor sino que también es un espacio de conflicto político, entonces la gestión pública debe incorporar esta dimensión en su quehacer diario; $y$, por otro lado, si nos situamos en un entorno democrático, esta incorporación no puede improvisarse sino que debe dar expresión a los valores democráticos del sistema político donde se ubica. Para mí sería suficiente con que al final del artículo quedara clara esta idea. Del mismo modo, debería quedar claro que, en este ámbito, los esfuerzos realizados hasta el momento por los teóricos de la gestión pública son bastante deficientes y que, en consecuencia, deberíamos potenciar nuestra reflexión en esta dirección.

Se me objetará, muy bien, ya bemos aceptado que es importante democratizar la gestión pública, pero ¿cómo se bace? ¿cómo podemos democratizar unas estructuras tan inercialmente dedicadas a la ejecución, tan cerradas sobre sus propia reglas y tan carentes de sensibilidad politica?. Evidentemente este artículo no responde a tales preguntas. Tan solo me remito a algu- nas propuestas (especialmente las de RANSON y STEWART), reclamo la necesidad de pensarlas y, en cualquier caso, considero que reconocer los límites de lo realizado hasta el momento y explicitar el problema supone un punto de partida esencial. Alguien puede decir que en lugar de empezar por una pregunta y acabar con una respuesta - que sería lo lógico-, empiezo con no se sabe muy bien qué y acabo con un interrogante. Pues sí, y ya me conformo.

¿De qué no hemos hablado? En primer lugar, tal como anticipo en el párrafo anterior, no hemos hablado sobre cómo democratizar las estructuras administrativas. Este es el tema realmente importante, ya que de nada sirve defender la democratización de la administración sin saber como llevarla a cabo. A pesar de tratarse del asunto clave, este artículo no lo aborda y se marca unos objetivos mucho más modestos. En cualquier caso, algunos trabajos apuntan que la democratización de la administración debería pasar por la introducción del discurso político y de las voces de los ciudadanos (que no de los clientes) en sus rutinas de funcionamiento. A partir de aquí, las propuestas giran alrededor de dos ejes: el papel de los líderes y la participación de los ciudadanos. Son ejes problemáticos, ya que a menudo pueden ser interpretados como trabas a la dimensión más instrumental de la administración; pero creo que su articulación se hará cada vez más inevitable. ¿Cómo? Pues aún no lo sabemos con exactitud, aunque sí sabemos que ésta es una línea de trabajo cada vez más importante en el campo de la gestión pública.

Aparte de frustrar las expectativas asociadas al cómo llevar a la práctica la democratización de la administración pública, también aparecen muchos otros asuntos que inevitablemente se entrecruzan en este tipo de discusiones y de los que no nos hemos ocupado. A uno de ellos quisiera referirme explícitamente, sobre todo para evitar posibles interpretaciones sesgadas: no hemos hablado de los programas de mejora de la eficiencia y la eficacia del funcionamiento administrativo. Es decir, cuando afirmo que la mayor parte de los teóricos de la nueva gestión pública tienden a olvidar la dimensión democrática, no estoy rechazando sus propuestas de forma global. Pueden ser altamente positivas en el ámbito de la mejora de los procedimientos internos y, de hecho, la mayoría son convincentes; pero yo no me he fijado en ellas sino en otra cosa. Lo que sí es cierto es que las considero demasiado dependientes del discurso que se elabora en la gestión privada y que, por lo tanto, olvidan las peculiaridades del ámbito público. Parafraseando el título de un artículo de METCALfE, hasta ahora la gestión pública se ha dedicado a imitar, se trata de empezar a innovar. 
- Universitat Autònoma de Barcelona.

\section{Bibliografia}

BaRzelay, M. (1992), Breaking Through Bureaucracy: A New Vision For Managing in Government, Berkeley, University of California Press.

Bauman, Z. (1989), Modernity and the Holocaust, Nueva York, Cornell University Press. BruGú, Q., Gomì, R. y Amoros, M. (1995), ·La Administración Pública y sus Clientes, Gestión y Análisis de Politicas Públicas, 꾸 1.

DruCKer, P. (1988), ·The Coming of the New Organization - Harvard Business Review, 88 (1).

Dunleavy, P. y Hood, C. (1995), .De la Administración Pública Tradicional a la Nueva Gestión Pública., Gestión y Análisis de Políticas Públicas, $n^{2} 3$.

DUNSIRE, A. (1993), Modes of Governance en Kooiman, J. (ed.) Modern Governance: New Government-Society Interactions, Londres, Sage.

Gomï, R. y Brugué, Q. (1995), ·Nous Models de Gestió i d'Organització Pública•, Autonomies, $n^{2} 8$.

Heckscher, C. (1994), -Defining the Post-Bureaucratic Type- en Heckscher,C. y Donnellon,A. (eds.) The Post-Bureaucratic Organization: New Perspectives on Organizational Change, Thousand Oaks, Sage.

Hirschman, A. O. (1970), Exit, Voice and Loyalty: Responses to Decline in Firms, Organisations and States, Cambridge, Harvard University Press.

Hood, C. (1994), Explaining Economic Policy Reversals, Buckingham, Open University Press.

Hughes, O. E. (1994), Public Management and Administration, Londres, Macmillan Press.
MetCalfe, L. y RichardS, S. (1989), La Modernización de la Gestión Püblica, Madrid, MAP.

METCALFE, L. (1993), Public Management: From Imitation to Innovatio- en Kooiman J. (ed.) Modern Governance, Londres, Sage.

Osborne, D. y GAEBler, T. (1994), La Reinvención del Gobierno: La Influencia del Espiritu Empresarial en el Sector Público, Barcelona, Paidós.

Peters, T. J. y Waterman, R. H. (1982), In Search of Excellence: Lessons from America's Best-Run Companies, Nueva York, Harper \& Row.

PolutT, C. (1993), Managerialism and the Public Services, Londres, Blackwell Publishers.

Ranson, S. y Stewart, J. (1994), Management for the Public Domain, Londres, Macmillan Press.

RITzER, G. (1996), The McDonaldization of Society, Thousand Oaks, Pine Forge Press.

Ritzer, G. y LEMOYNE, T. (1991), •Hyperrationality• en Ritzer,G. (ed.) Methatbeorizing in Sociology, Lexington, Lexington Books.

SCHEIN, E. H. (1985), Organizational Culture and Leadership, San Francisco, JosseyBass.

Schwarz, M. y Thompson, M. (1990), Divided We Stand, Hemel Hempstead, Harvester Whearsheaf.

TAKAKI, R. (1990), Iron Cages: Races and Culture in 19th-Century America, Nueva York, Oxford University Press. 